\title{
The Grassmannian for celestial superamplitudes
}

\author{
Livia Ferro $^{a, b}$ and Robert Moerman ${ }^{b}$ \\ ${ }^{a}$ Arnold-Sommerfeld-Center for Theoretical Physics, Ludwig-Maximilians-Universität, \\ Theresienstraße 37, München 80333, Germany \\ ${ }^{b}$ Department of Physics, Astronomy and Mathematics, University of Hertfordshire, \\ College Lane, Hatfield AL10 9AB, U.K. \\ E-mail: livia.ferro@lmu.de, r.moerman@herts.ac.uk
}

ABstract: Recently, scattering amplitudes in four-dimensional Minkowski spacetime have been interpreted as conformal correlation functions on the two-dimensional celestial sphere, the so-called celestial amplitudes. In this note we consider tree-level scattering amplitudes in $\mathcal{N}=4$ super Yang-Mills theory and present a Grassmannian formulation of their celestial counterparts. This result paves the way towards a geometric picture for celestial superamplitudes, in the spirit of positive geometries.

Keywords: Scattering Amplitudes, Supersymmetric Gauge Theory

ArXIV EPrint: 2107.07496 


\section{Contents}

1 Introduction 1

2 Definitions 2

2.1 Celestial amplitudes 2

2.2 Grassmannian integrals 3

3 Grassmannian on the celestial sphere 4

3.1 Examples 6

\section{Introduction}

In the traditional momentum space, amplitudes are specified by asymptotic wave functions, which transform simply under spacetime translations, and describe transitions between momentum eigenstates. But what if four-dimensional Minkowski space is not the right space to see all their properties? Very recently, attention has surged towards celestial amplitudes. For the massless case, celestial amplitudes are the Mellin transform of scattering amplitudes in four-dimensional Minkowski space and are conformal correlation functions on the two-dimensional celestial sphere, i.e. the null infinity of four-dimensional Minkowski space. This map was proposed in [1], and explicitly applied to three and four-point treelevel gluon amplitudes in [2] for the first time. It stems from the observation that there is a basis which represents massless spin $s$ free fields in four-dimensional Minkowski space as spin $s$ conformal primaries on the two-sphere. Indeed, the Lorentz group $\mathrm{SO}(1,3)$ is mapped to the two-dimensional conformal group $\mathrm{SL}(2, \mathbb{C})$ : therefore, scattering amplitudes (i.e. momentum eigenstates) in four-dimensional Minkowski space can be interpreted as two-dimensional conformal correlators (i.e. boosts eigenstates) of primary fields. Since its inception, a lot of progress has been made: in the computation of the Mellin transform of various tree-level gluon amplitudes [3], of all-loop four-point amplitudes [4], and of string amplitudes [5]; in the study of symmetries and soft theorems [6-16]; in extensions to supersymmetric theories [17-20].

On one side, understanding the precise nature of the two-dimensional conformal field theory on the celestial sphere would enable a holographic description of spacetime. On the other, celestial amplitudes provide us with yet another powerful tool for exploring the mathematical properties of ordinary amplitudes by using our knowledge of conformal field theories. However, our understanding is still preliminary and more work is necessary to advance it to the same level as, or even beyond, our understanding of ordinary amplitudes. We have only started to walk the same path which we travelled for understanding ordinary amplitudes - analytic properties, factorization, symmetries - nevertheless, we do not know anything about their geometric structure. The natural question which arises is 
whether we can learn more about scattering amplitudes, and therefore further constrain the S-matrix, by understanding of the conformal field theory on the celestial sphere. Moreover, does a positive geometry [21] for celestial amplitudes exist?

In this paper we start to fill this gap by presenting a Grassmannian formulation of celestial superamplitudes. A first look at Grassmannian formulae for amplitudes has been presented in [22], where tree-level gluon amplitudes have been considered. We want to go beyond this and consider the supersymmetric version. Indeed, if we want to retrace the path we have covered for ordinary amplitudes in momentum space, this is the first step towards the definition of (positive) geometries for celestial amplitudes. In particular, the results of $[23,24]$, which presented tree-level scattering amplitudes in $\mathcal{N}=4$ super YangMills (sYM) theory in terms of integrals over a Grassmannian space, paved the way for the discovery of the amplituhedron [25] and, more generally, positive geometries underlying scattering amplitudes in various theories. Therefore, we consider scattering amplitudes in

$\mathcal{N}=4$ sYM. This theory enjoys a high amount of symmetry, the infinite-dimensional Yangian symmetry [26], which is beautifully realized in the Grassmannian formulation [27, 28]. This feature of the theory makes it simpler to explore and therefore a useful playground to investigate properties of amplitudes. Moreover, while all previous explicit results for celestial amplitudes were either worked out on a case-by-case basis or resulted in very involved expressions, a Grassmannian formula is usually very neat and renders some properties more manifest. To this end, in this note we perform the Mellin transform of the Grassmannian integral representation of the $n$-point helicity $k$ tree-level superamplitude and thereby find its celestial counterpart. This is the long sought-after Grassmannian formulation of celestial superamplitudes and the starting point for a plethora of investigations which are expected to bring us novel information both on the holography side and on the ordinary amplitudes side.

This note is organised as follows. In the next section we review some definitions which will be useful for presenting our main result. In particular, we will review celestial amplitudes and the Grassmannian formulation of scattering amplitudes in $\mathcal{N}=4$ sYM. Thereafter we perform the Mellin transform of the Grassmannian formulation and find its celestial version. We then present some examples.

\section{Definitions}

In this section we briefly review some of the definitions that will be needed for the main result of this note. In particular, we review the celestial (super)amplitudes and the Grassmannian formulation of scattering amplitudes in $\mathcal{N}=4 \mathrm{sYM}$.

\subsection{Celestial amplitudes}

In the following we will consider tree-level scattering amplitudes of massless particles. The massless momenta can be then written in terms of the spinor-helicity variables $p^{\alpha \dot{\alpha}}=\lambda^{\alpha} \tilde{\lambda}^{\dot{\alpha}}$, $\alpha, \dot{\alpha}=1,2$. To map the amplitudes from Minkowski space to the celestial sphere, we first need to introduce celestial coordinates and parametrize the massless momenta in the 
following way:

$$
p^{\alpha \dot{\alpha}}=\epsilon w k^{\alpha \dot{\alpha}}
$$

where $w$ is the angular frequency associated to the energy of the particle, conventionally called "energy", and $k^{\alpha \dot{\alpha}}=\xi^{\alpha} \tilde{\xi}^{\dot{\alpha}}$ with

$$
\xi^{\alpha}=\left(\begin{array}{c}
z \\
1
\end{array}\right), \quad \tilde{\xi}^{\dot{\alpha}}=\left(\begin{array}{c}
\bar{z} \\
1
\end{array}\right),
$$

where $z, \bar{z}$ are the coordinates on the celestial sphere. $\epsilon$ is a sign which depends on whether the particle is incoming or outgoing. We parametrise the spinors as

$$
\lambda^{\alpha}=\epsilon \sqrt{w}\left(\begin{array}{c}
z \\
1
\end{array}\right)=\epsilon \sqrt{w} \xi^{\alpha}, \quad \tilde{\lambda}^{\dot{\alpha}}=\sqrt{w}\left(\begin{array}{c}
\bar{z} \\
1
\end{array}\right)=\sqrt{w} \tilde{\xi}^{\dot{\alpha}} .
$$

Since we are working with superamplitudes, we also have Grassmann variables $\eta^{A} \mathrm{~s}$. In the language of celestial amplitudes, they will be called $\tilde{\tau}^{A}[19]$. After performing this change of variables, the massless scattering superamplitude is mapped on the celestial sphere via a Mellin transform [18, 19]:

$$
\tilde{A}_{n, k}\left(\left\{\Delta_{i}, z_{i}, \bar{z}_{i}, \tilde{\tau}_{i}\right\}\right)=\prod_{i=1}^{n} \int_{0}^{\infty} d w_{i} w_{i}^{\Delta_{i}-1} A_{n, k}\left(\left\{w_{i}, z_{i}, \bar{z}_{i}, \tilde{\tau}_{i}\right\}\right),
$$

where the celestial superamplitude transforms as a two-dimensional conformal correlator on the celestial sphere with weights $\Delta_{i}$. We end this section by remarking that, under conformal transformations, we have the following behaviour

$w \rightarrow w^{\prime}=|c z+d|^{2} w, \quad z \rightarrow z^{\prime}=\frac{a z+b}{c z+d}, \quad \bar{z} \rightarrow \bar{z}^{\prime}=\frac{\bar{a} \bar{z}+\bar{b}}{\bar{c} \bar{z}+\bar{d}}, \quad \tilde{\tau}^{A} \rightarrow \tilde{\tau}^{\prime A}=\frac{(c z+d)^{1 / 2}}{(\bar{c} \bar{z}+\bar{d})^{1 / 2}} \tilde{\tau}^{A}$,

with $a, b, c, d \in \mathbb{C}$ and $a d-b c=1$.

\subsection{Grassmannian integrals}

Let us now review the formulation of scattering amplitudes in $\mathcal{N}=4 \mathrm{sYM}$ in terms of Grassmannian integrals. In $[23,24]$ the leading singularities of the $\mathcal{N}=4 \mathrm{sYM} \mathrm{N}^{k-2} \mathrm{MHV}$ $n$-point amplitudes were described by an integral over the space of $k$-planes in $n$ dimensions, the Grassmannian $G(k, n)$, along suitable closed contours. This arose from the observation that momentum conservation, which is a quadratic constraint in the spinor-helicity space, can be linearised by using auxiliary spaces. Indeed, we can introduce an auxiliary $k$-plane in $n$-dimensions, $C=\left(c_{a i}\right)$, such that

$$
C \cdot \tilde{\lambda}=0, \quad \lambda \cdot C^{\perp}=0,
$$

where $C^{\perp}$ is the orthogonal complement of $C$. In this way, the condition $\sum_{i=1}^{n} \lambda_{i}^{\alpha} \widetilde{\lambda}_{i}^{\dot{\alpha}}=0$ is linearised. The tree-level amplitudes in spinor-helicity space can therefore be written as

$$
A_{n, k}=\int_{\gamma} d \Omega_{n, k} \prod_{\dot{a}=1}^{k} \delta^{2}\left(\sum_{i=1}^{n} c_{\dot{a} i} \tilde{\lambda}_{i}\right) \prod_{a=k+1}^{n} \delta^{2}\left(\sum_{i=1}^{n} c_{a i}^{\perp} \lambda_{i}\right) \prod_{\dot{a}=1}^{k} \delta^{4}\left(\sum_{i=1}^{n} c_{\dot{a} i} \eta_{i}\right),
$$


where $\gamma$ is a closed contour. The measure is

$$
d \Omega_{n, k}=\frac{\prod_{\dot{a}, i} d c_{\dot{a} i}}{\operatorname{GL}(k)(1 \ldots k)(2 \ldots k+1) \ldots(n \ldots n+k-1)}
$$

where the denominator consists of the cyclic product of the minors $\mathcal{M}_{i}=(i i+1 \ldots i+k-1)$, i.e. the determinants of $(k \times k)$ submatrices of the matrix $C$. The contour $\gamma$ can be determined by using e.g. the Britto-Cachazo-Feng-Witten (BCFW) recursion relations [29, 30].

\section{Grassmannian on the celestial sphere}

We are now ready to consider our scattering superamplitude in the Grassmannian formulation (2.7) and perform the Mellin transform. Despite the simplicity of this idea, we will soon discover that in the process a few tricks need to be used in order to find a neat result. We start by using the $\mathrm{GL}(k)$ redundancy to write $C \in G(k, n)$ as

$$
C_{\dot{a} \dot{b}}=\delta_{\dot{a} \dot{b}}, \quad C_{\dot{a} b}=c_{\dot{a} b} .
$$

With this particular choice, the bosonic delta-functions in (2.7) read

$$
\delta^{k \times 2}(C \cdot \tilde{\lambda})=\prod_{\dot{a}} \delta^{2}\left(\tilde{\lambda}_{\dot{a}}+\sum_{b} c_{\dot{a} b} \tilde{\lambda}_{b}\right), \quad \delta^{2 \times(n-k)}\left(\lambda \cdot C^{\perp}\right)=\prod_{a} \delta^{2}\left(\lambda_{a}-\sum_{\dot{b}} c_{\dot{b} a} \lambda_{\dot{b}}\right) .
$$

If we now move to celestial coordinates, these delta-functions will contain square roots of the form $\sqrt{\omega_{i}}$ introduced via (2.3). To remove these and render the computation easier, we utilize the trick presented in [22] and use the little group scaling transformations

$$
\lambda_{i} \rightarrow t_{i} \lambda_{i}, \quad \tilde{\lambda}_{i} \rightarrow t_{i}^{-1} \tilde{\lambda}_{i}, \quad \tilde{\eta}_{i} \rightarrow t_{i}^{-1} \tilde{\eta}_{i},
$$

which are reflected on the superamplitude as $A_{n} \rightarrow t_{i}^{2} A_{n}$. In particular, we choose different forms for the scalings of different particles:

$$
t_{\dot{a}}=w_{\dot{a}}^{-1 / 2}, \dot{a}=1, \ldots, k, \quad t_{a}=w_{a}^{1 / 2}, a=k+1, \ldots, n .
$$

After performing this little group transformation, we find that

$$
\begin{aligned}
& \tilde{A}_{n, k}=\int_{\gamma} d \Omega_{n, k}\left(\prod_{i=1}^{n} \int_{0}^{\infty} \frac{d w_{i}}{w_{i}} w_{i}^{\Delta_{i}}\right) \prod_{\dot{a}} w_{\dot{a}}^{-1} \prod_{a} w_{a} \\
& \cdot \prod_{\dot{a}} \delta^{2}\left(w_{\dot{a}} \tilde{\xi}_{\dot{a}}+\sum_{b} c_{\dot{a} b} \tilde{\xi}_{b}\right) \prod_{a} \delta^{2}\left(\epsilon_{a} w_{a} \xi_{a}-\sum_{\dot{b}} c_{\dot{b} a} \epsilon_{\dot{b}} \xi_{\dot{b}}\right) \prod_{\dot{a}} \delta^{4}\left(\sqrt{w_{\dot{a}}} \tilde{\tau}_{\dot{a}}+\sum_{b} c_{\dot{a} b} \frac{\tilde{\tau}_{b}}{\sqrt{w_{b}}}\right),
\end{aligned}
$$

where now no square root is present in the bosonic delta-functions. To illuminate the transformational properties of $\tilde{A}_{n, k}$, it will prove useful to introduce auxiliary parameters 
$p_{i}$ and $\bar{p}_{i}$ for each particle $i$, where $\bar{p}_{i}=\left(p_{i}\right)^{*}$ or both parameters are real and independent. Rewriting $w_{i}$ as

$$
w_{i} \equiv\left(p_{i} \bar{p}_{i}\right)^{2} y_{i}
$$

we find

$$
\begin{aligned}
\tilde{A}_{n, k}= & \prod_{i=1}^{n}\left[\left(p_{i} \bar{p}_{i}\right)^{2}\right]^{\Delta_{i}} \prod_{\dot{a}} \frac{p_{\dot{a}}^{2}}{\bar{p}_{\dot{a}}^{6}} \prod_{a} \frac{1}{\left(p_{a} \bar{p}_{a}\right)^{2}} \int_{\gamma} d \Omega_{n, k}\left(\prod_{i=1}^{n} \int_{0}^{\infty} d y_{i} y_{i}^{\Delta_{i}}\right) \\
& \cdot \prod_{\dot{a}} \delta^{2}\left(y_{\dot{a}} \tilde{\xi}_{\dot{a}}+\sum_{b} c_{\dot{a} b} \frac{\tilde{\xi}_{b}}{\left(p_{\dot{a}} \bar{p}_{\dot{a})^{2}}\right.}\right) \prod_{a} \delta^{2}\left(\epsilon_{a} y_{a} \xi_{a}-\sum_{\dot{b}} c_{\dot{b} a} \frac{\epsilon_{\dot{b}} \xi_{\dot{b}}}{\left(p_{a} \bar{p}_{a}\right)^{2}}\right) \\
& \cdot \prod_{\dot{a}} \delta^{4}\left(\tau_{\dot{a}}+\sum_{b} \frac{c_{\dot{a} b}}{\left(p_{\dot{a}} \bar{p}_{b}\right)^{2}} \frac{\tau_{b}}{\sqrt{y_{\dot{a}}} \sqrt{y_{b}}}\right)
\end{aligned}
$$

where we have defined new fermionic variables

$$
\tau_{i} \equiv \frac{\bar{p}_{i}}{p_{i}} \tilde{\tau}_{i}
$$

By performing the change of variables ${ }^{1} c_{\dot{a} b} \rightarrow c_{\dot{a} b}\left(p_{\dot{a}} \bar{p}_{b}\right)^{2}$, the bosonic delta-functions can then be written as

$$
\begin{aligned}
\delta^{2}\left(y_{\dot{a}} \tilde{\xi}_{\dot{a}}+\sum_{b} c_{\dot{a} b} \frac{\tilde{\xi}_{b}}{\left(p_{\dot{a}} \bar{p}_{\dot{a}}\right)^{2}}\right) & \rightarrow \bar{p}_{\dot{a}}^{4} \delta\left(y_{\dot{a}}-E_{\dot{a}}\right) \delta\left(\sum_{b} c_{\dot{a} b}\left(\bar{p}_{\dot{a}} \bar{p}_{b}\right)^{2} \bar{z}_{\dot{a} b}\right), \\
\delta^{2}\left(\epsilon_{a} y_{a} \xi_{a}-\sum_{\dot{b}} c_{\dot{b} a} \frac{\epsilon_{\dot{b}} \xi_{\dot{b}}}{\left(p_{a} \bar{p}_{a}\right)^{2}}\right) & \rightarrow p_{a}^{4} \delta\left(y_{a}-E_{a}\right) \delta\left(\sum_{\dot{b}} c_{\dot{b} a}\left(p_{\dot{b}} p_{a}\right)^{2} \epsilon_{\dot{b}} \epsilon_{a} z_{\dot{b} a}\right),
\end{aligned}
$$

where

$$
E_{\dot{a}}=-\sum_{b} c_{\dot{a} b}\left(\frac{\bar{p}_{b}}{\bar{p}_{\dot{a}}}\right)^{2} \frac{[b \tilde{\chi}]}{[\dot{a} \tilde{\chi}]}, \quad E_{a}=\sum_{\dot{b}} c_{\dot{b} a}\left(\frac{p_{\dot{b}}}{p_{a}}\right)^{2} \frac{\epsilon_{\dot{b}}\langle\dot{b} \chi\rangle}{\epsilon_{a}\langle a \chi\rangle}
$$

are "energies" as in [22] and $\chi^{\alpha}, \tilde{\chi}^{\dot{\alpha}}$ are auxiliary reference spinors. Finally, we obtain the main result of our paper: the Mellin transform of the $\mathcal{N}=4 \mathrm{sYM}$ amplitudes in the Grassmannian formulation is

$$
\tilde{A}_{n, k}=P_{n} \cdot I_{n, k},
$$

where $I_{n, k}$ is an integral over the Grassmannian $G(k, n)$

$$
\begin{aligned}
I_{n, k}= & \int_{\gamma} d \Omega_{n, k} \prod_{i=1}^{n} E_{i}^{\Delta_{i}} \mathbb{1}_{>0}\left(E_{i}\right) \\
& \cdot \prod_{\dot{a}} \delta\left(\sum_{b} c_{\dot{a} b}\left(\bar{p}_{\dot{a}} \bar{p}_{b}\right)^{2} \bar{z}_{\dot{a} b}\right) \prod_{a} \delta\left(\sum_{\dot{b}} c_{\dot{b} a}\left(p_{\dot{b}} p_{a}\right)^{2} \epsilon_{\dot{b}} \epsilon_{a} z_{\dot{b} a}\right) \prod_{\dot{a}} \delta^{4}\left(\tau_{\dot{a}}+\sum_{b} c_{\dot{a} b} \frac{\tau_{b}}{\sqrt{E_{\dot{a}} E_{b}}}\right),
\end{aligned}
$$

\footnotetext{
${ }^{1}$ This change of variables leaves the Grassmannian measure $d \Omega_{n, k}$ invariant.
} 
and $P_{n} \equiv\left(\prod_{i=1}^{n} p_{i}^{2\left(\Delta_{i}+1\right)} \bar{p}_{i}^{2\left(\Delta_{i}-1\right)}\right)$. The indicator function $\mathbb{1}_{>0}\left(E_{i}\right)$ is defined to be one if $E_{i}$ is real and non-negative, and zero otherwise. It appears because of the integration ranges for $w$ 's are the positive real line.

If we now specify that, under a conformal transformation, $p_{i}$ and $\bar{p}_{i}$ transform according to

$$
p_{i} \rightarrow\left(c z_{i}+d\right)^{1 / 2} p_{i}, \quad \bar{p}_{i} \rightarrow\left(\bar{c} \bar{z}_{i}+\bar{d}\right)^{1 / 2} \bar{p}_{i}
$$

then the Grassmannian integral $I_{n, k}$ defined above is conformally invariant, while the prefactor $P_{n, k}$ transforms covariantly

$$
P_{n} \rightarrow\left(\prod_{i=1}^{n}\left(c z_{i}+d\right)^{\Delta_{i}+1}\left(\bar{c} \bar{z}_{i}+\bar{d}\right)^{\Delta_{i}-1}\right) P_{n} .
$$

Altogether, this ensures that $\tilde{A}_{n, k}$ transforms as expected under conformal transformations. Thus, the auxiliary parameters $p_{i}$ and $\bar{p}_{i}$ make the transformation properties of $\tilde{A}_{n, k}$ manifest.

One could, however, make any choice for $p_{i}$ and $\bar{p}_{i}$ in order to evaluate $\tilde{A}_{n, k}$, and the result would be the same, but the transformation properties of $\tilde{A}_{n, k}$ might not be manifest. For example, choosing $p_{i}=\bar{p}_{i}=1$, we obtain the Grassmannian integral

$$
\begin{aligned}
A_{n, k}= & \int_{\gamma} d \Omega_{n, k} \prod_{i=1}^{n} E_{i}^{\Delta_{i}} \mathbb{1}_{>0}\left(E_{i}\right) \\
& \cdot \prod_{\dot{a}} \delta\left(\sum_{b} c_{\dot{a} b} \bar{z}_{\dot{a} b}\right) \prod_{a} \delta\left(\sum_{\dot{b}} c_{\dot{b} a} \epsilon_{\dot{b}} \epsilon_{a} z_{\dot{b} a}\right) \prod_{\dot{a}} \delta^{4}\left(\tilde{\tau}_{\dot{a}}+\sum_{b} c_{\dot{a} b} \frac{\tilde{\tau}_{b}}{\sqrt{E_{\dot{a}} E_{b}}}\right),
\end{aligned}
$$

where $\tilde{\tau}_{i}$ are the original fermionic parameters and the energies read

$$
E_{\dot{a}}=-\sum_{b} c_{\dot{a} b} \frac{[b \tilde{\chi}]}{[\dot{a} \tilde{\chi}]}, \quad E_{a}=\sum_{\dot{b}} c_{\dot{b} a} \frac{\epsilon_{\dot{b}}\langle\dot{b} \chi\rangle}{\epsilon_{a}\langle a \chi\rangle} .
$$

We have checked that formula (3.17) correctly reproduces the formula (6.13) in [22] when projected onto pure gluon amplitudes.

\subsection{Examples}

Three-point MHV. Let us consider the case $(n, k)=(3,2)$ with $\epsilon_{1}=\epsilon_{2}=-\epsilon_{3}=1$. Since this is the simplest case, we present it in full detail, with most calculations explicit. In Minkowski signature the on-shell three-point amplitude vanishes. Thus, we assume to be working in the $(2,2)$ split signature, where $z_{i}$ and $\bar{z}_{i}$ are real, independent variables. Consider the following gauge fixing

$$
C=\left(\begin{array}{ccc}
1 & 0 & c_{13} \\
0 & 1 & -c_{23}
\end{array}\right)
$$


where the minus sign is chosen for convenience. Consider, first, the bosonic delta-functions in (3.16). They are explicitly

$$
\prod_{\dot{a}} \delta\left(\sum_{b} c_{\dot{a} b} \bar{z}_{\dot{a} b}\right) \prod_{a} \delta\left(\sum_{\dot{b}} c_{\dot{b} a} \epsilon_{\dot{b}} \epsilon_{a} z_{\dot{b} a}\right)=c_{13}^{-1} c_{23}^{-1} z_{31}^{-1} \delta\left(\bar{z}_{23}\right) \delta\left(\bar{z}_{31}\right) \delta\left(c_{13}+c_{23} \frac{z_{23}}{z_{31}}\right) .
$$

On the support of the last delta-function, the energies become

$$
E_{1}=c_{23} \frac{z_{23}}{z_{31}}, \quad E_{2}=c_{23}, \quad E_{3}=c_{23} \frac{z_{21}}{z_{31}},
$$

and consequently, the indicator functions simplify to

$$
\prod_{i=1}^{3} \mathbb{1}_{>0}\left(E_{i}\right)=\mathbb{1}_{>0}\left(c_{23}\right) \Theta\left(\frac{z_{21}}{z_{23}}\right) \Theta\left(\frac{z_{21}}{z_{31}}\right)
$$

where $\Theta$ is the Heaviside step function. Notice that $\mathbb{1}_{>0}\left(c_{23}\right)$ restricts the integral for $c_{23}$, which was originally a complex integral, to the positive reals. The fermionic delta-functions in (3.16) simplify to

$$
\begin{aligned}
\prod_{\dot{a}} \delta^{4}\left(\tau_{\dot{a}}+\sum_{b} c_{\dot{a} b} \frac{\tau_{b}}{\sqrt{E_{\dot{a}} E_{b}}}\right) & =\delta^{4}\left(\tilde{\tau}_{1}-\sqrt{\frac{z_{23}}{z_{21}}} \tilde{\tau}_{3}\right) \delta^{4}\left(\tilde{\tau}_{2}-\sqrt{\frac{z_{31}}{z_{21}}} \tilde{\tau}_{3}\right) \\
& =z_{23}^{-2} z_{31}^{-2} z_{12}^{-4} \delta^{8}\left(\sqrt{z_{23}} \xi_{1} \tilde{\tau}_{1}+\sqrt{z_{31}} \xi_{2} \tilde{\tau}_{2}-\sqrt{z_{21}} \xi_{3} \tilde{\tau}_{3}\right)
\end{aligned}
$$

Combining all of these simplifications, one obtains

$$
\begin{aligned}
\tilde{A}_{3,2}= & z_{12}^{\Delta_{3}-4} z_{23}^{\Delta_{1}-4} z_{31}^{-1-\Delta_{1}-\Delta_{3}} \delta\left(\bar{z}_{23}\right) \delta\left(\bar{z}_{31}\right) \Theta\left(\frac{z_{21}}{z_{23}}\right) \Theta\left(\frac{z_{21}}{z_{31}}\right) \\
& \cdot \delta^{8}\left(\sqrt{z_{23}} \xi_{1} \tilde{\tau}_{1}+\sqrt{z_{31}} \xi_{2} \tilde{\tau}_{2}-\sqrt{z_{21}} \xi_{3} \tilde{\tau}_{3}\right) \int_{0}^{\infty} \frac{d c_{23}}{c_{23}} c_{23}^{\left(\Delta_{1}+\Delta_{2}+\Delta_{3}\right)-3} .
\end{aligned}
$$

Writing $\Delta_{i}=i \beta_{i}+1$ for $\beta_{i} \in \mathbb{R}$ and defining $\beta \equiv \beta_{1}+\beta_{2}+\beta_{3}$, the remaining integral simplifies to

$$
\int_{0}^{\infty} \frac{d c_{23}}{c_{23}} c_{23}^{\left(\Delta_{1}+\Delta_{2}+\Delta_{3}\right)-3}=2 \pi \delta(\beta),
$$

and on the support of this delta-function, the three-point MHV celestial superamplitude is given by

$$
\begin{aligned}
\tilde{A}_{3,2}= & 2 \pi \delta(\beta) z_{12}^{\Delta_{3}-4} z_{23}^{\Delta_{1}-4} z_{31}^{\Delta_{2}-4} \delta\left(\bar{z}_{23}\right) \delta\left(\bar{z}_{31}\right) \Theta\left(\frac{z_{21}}{z_{23}}\right) \Theta\left(\frac{z_{21}}{z_{31}}\right) \\
& \cdot \delta^{8}\left(\sqrt{z_{23}} \xi_{1} \tilde{\tau}_{1}+\sqrt{z_{31}} \xi_{2} \tilde{\tau}_{2}-\sqrt{z_{21}} \xi_{3} \tilde{\tau}_{3}\right)
\end{aligned}
$$

in agreement with $[18,19]$.

The three-point $\overline{\mathrm{MHV}}$ celestial superamplitude can be calculated in a similar way, where this time the gauge-fixed $C$-matrix reads $C=\left(\begin{array}{lll}1 & c_{13} & c_{23}\end{array}\right)$. 
Four-point MHV. For the case $(n, k)=(4,2)$, and $\epsilon_{1}=\epsilon_{2}=-\epsilon_{3}=-\epsilon_{4}=1$, we only present a few features as the computation is involved and tedious, nevertheless it can be performed straightforwardly. In this case the matrix $C$ reads

$$
C=\left(\begin{array}{llll}
1 & 0 & c_{13} & c_{14} \\
0 & 1 & c_{23} & c_{24}
\end{array}\right) \text {. }
$$

The bosonic delta-functions give the following expression

$$
\begin{aligned}
& \prod_{\dot{a}} \delta\left(\sum_{b} c_{\dot{a} b} \bar{z}_{\dot{a} b}\right) \prod_{a} \delta\left(\sum_{\dot{b}} c_{\dot{b} a} \epsilon_{\dot{b}} \epsilon_{a} z_{\dot{b} a}\right) \\
& =\frac{1}{c_{24}\left|z_{13} z_{24}\right|^{2}} \delta(r-\bar{r}) \delta\left(c_{13}+c_{24}(r-1) \frac{\left|z_{24}\right|^{2}}{z_{14} \bar{z}_{23}}\right) \delta\left(c_{14}+c_{24} \frac{z_{24}}{z_{14}}\right) \delta\left(c_{23}+c_{24} \frac{\bar{z}_{24}}{\bar{z}_{23}}\right),
\end{aligned}
$$

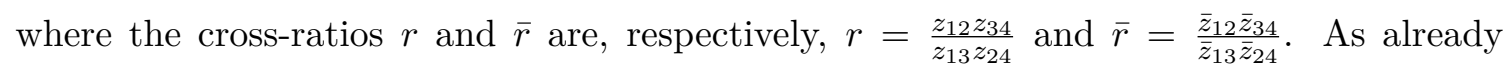
noticed in, e.g. [2], the factor $\delta(r-\bar{r})$ ensures that in the four-particle scattering the momenta of all particles lie on the same plane. The intersection of this plane with the celestial sphere forces the cross-ratio $r$ to be real. The energies on the support of the above delta-functions become

$$
E_{1}=(r-1) \frac{\left|z_{24}\right|^{2}}{\left|z_{14}\right|^{2}} E_{2}=\tilde{c}_{24}(r-1) \frac{\left|z_{24}\right|^{2}}{\left|z_{14}\right|^{2}}, \quad E_{3}=(r-1) \frac{\left|z_{24}\right|^{2}}{\left|z_{23}\right|^{2}} E_{4}=\tilde{c}_{24} r \frac{\left|z_{24}\right|^{2}}{\left|z_{34}\right|^{2}},
$$

where $\tilde{c}_{24}=c_{24} \frac{\bar{z}_{34}}{\bar{z}_{23}}$. In particular, $E_{2}=\tilde{c}_{24}$. The indicator functions require these energies to be real and positive which forces $r>1$, producing $\Theta(r-1)$, and $\tilde{c}_{24}$ to be real and positive, producing $\mathbb{1}_{>0}\left(\tilde{c}_{24}\right)$. With a little effort, one can show that the fermionic deltafunctions can be written as

$$
\begin{aligned}
& \prod_{\dot{a}} \delta^{4}\left(\tilde{\tau}_{\dot{a}}+\sum_{b} c_{\dot{a} b} \frac{\tilde{\tau}_{b}}{\sqrt{E_{\dot{a}} E_{b}}}\right) \\
& =z_{12}^{-4}\left|\frac{z_{23} z_{24}}{z_{13} z_{14}}\right|^{2} \delta^{8}\left(\xi_{1} \tilde{\tau}_{1}+\left|\frac{z_{13} z_{14}}{z_{23} z_{24}}\right|^{1 / 2} \xi_{2} \tilde{\tau}_{2}-\left|\frac{z_{12} z_{14}}{z_{23} z_{34}}\right|^{1 / 2} \xi_{3} \tilde{\tau}_{3}-\left|\frac{z_{12} z_{13}}{z_{24} z_{34}}\right|^{1 / 2} \xi_{4} \tilde{\tau}_{4}\right),
\end{aligned}
$$

and it is clearly independent of $\tilde{c}_{24}$. The product of all energies, each raised to the power of their respective weight, evaluates to

$$
\begin{aligned}
\prod_{i=1}^{4} E_{i}^{\Delta_{i}}= & (-1)^{\Delta_{1}+\Delta_{2}} \tilde{c}_{24}^{\Delta_{1}+\Delta_{2}+\Delta_{3}+\Delta_{4}}\left(\frac{z_{14} z_{23}}{z_{34}^{2}}\right)^{2}\left(\frac{\bar{z}_{12}^{2}}{\bar{z}_{14} \bar{z}_{23}}\right)^{2} \\
& \cdot\left(\frac{z_{13}}{\bar{z}_{42}}\right)^{\Delta_{2}+\Delta_{4}-4}\left(\frac{\bar{z}_{12}}{z_{34}}\right)^{\Delta_{3}+\Delta_{4}-4}\left(\frac{\bar{z}_{14}}{z_{23}}\right)^{\Delta_{2}-2}\left(\frac{z_{14}}{\bar{z}_{23}}\right)^{\Delta_{3}-2} .
\end{aligned}
$$

Collecting all factors of $\tilde{c}_{24}$ and integrating over all $\tilde{c}_{2}$ (on the support of $\mathbb{1}_{>0}\left(\tilde{c}_{24}\right)$ ) produces the delta-function

$$
\int_{0}^{\infty} \frac{d \tilde{c}_{24}}{\tilde{c}_{24}} \tilde{c}_{24}^{\left(\Delta_{1}+\Delta_{2}+\Delta_{3}+\Delta_{4}\right)-4}=2 \pi \delta(\beta)
$$


where, again, $\Delta_{i}=i \beta_{i}+1$ for $\beta_{i} \in \mathbb{R}$ and $\beta \equiv \beta_{1}+\beta_{2}+\beta_{3}+\beta_{4}$. Finally, the four-point MHV celestial superamplitude is given by

$$
\begin{aligned}
& \tilde{A}_{4,2}=2 \pi \delta(\beta)(-1)^{\Delta_{1}+\Delta_{2}}\left(\frac{z_{13}}{\bar{z}_{42}}\right)^{\Delta_{2}+\Delta_{4}-4}\left(\frac{\bar{z}_{12}}{z_{34}}\right)^{\Delta_{3}+\Delta_{4}-4}\left(\frac{\bar{z}_{14}}{z_{23}}\right)^{\Delta_{2}-2}\left(\frac{z_{14}}{\bar{z}_{23}}\right)^{\Delta_{3}-2} \\
& \cdot \frac{\delta(r-\bar{r}) \Theta(r-1)}{z_{12} z_{23} z_{34} z_{41}\left|z_{13}\right|^{2}\left|z_{24}\right|^{2}} \delta^{8}\left(\xi_{1} \tilde{\tau}_{1}+\left|\frac{z_{13} z_{14}}{z_{23} z_{24}}\right|^{1 / 2} \xi_{2} \tilde{\tau}_{2}-\left|\frac{z_{12} z_{14}}{z_{23} z_{34}}\right|^{1 / 2} \xi_{3} \tilde{\tau}_{3}-\left|\frac{z_{12} z_{13}}{z_{24} z_{34}}\right|^{1 / 2} \xi_{4} \tilde{\tau}_{4}\right),
\end{aligned}
$$

in agreement with [19].

\section{Acknowledgments}

We would like to thank Tomek Łukowski for useful comments on the draft. L.F. thanks David Damgaard for collaboration at early stages of this project. This work was partially funded by the Deutsche Forschungsgemeinschaft (DFG, German Research Foundation) Projektnummern 404358295 and 404362017.

Open Access. This article is distributed under the terms of the Creative Commons Attribution License (CC-BY 4.0), which permits any use, distribution and reproduction in any medium, provided the original author(s) and source are credited.

\section{References}

[1] C. Cheung, A. de la Fuente and R. Sundrum, $4 D$ scattering amplitudes and asymptotic symmetries from 2D CFT, JHEP 01 (2017) 112 [arXiv:1609.00732] [INSPIRE].

[2] S. Pasterski, S.-H. Shao and A. Strominger, Gluon Amplitudes as $2 d$ Conformal Correlators, Phys. Rev. D 96 (2017) 085006 [arXiv:1706.03917] [inSPIRE].

[3] A. Schreiber, A. Volovich and M. Zlotnikov, Tree-level gluon amplitudes on the celestial sphere, Phys. Lett. B $\mathbf{7 8 1}$ (2018) 349 [arXiv:1711.08435] [INSPIRE].

[4] H.A. González, A. Puhm and F. Rojas, Loop corrections to celestial amplitudes, Phys. Rev. D 102 (2020) 126027 [arXiv:2009.07290] [INSPIRE].

[5] S. Stieberger and T.R. Taylor, Strings on Celestial Sphere, Nucl. Phys. B 935 (2018) 388 [arXiv: 1806. 05688] [INSPIRE].

[6] L. Donnay, A. Puhm and A. Strominger, Conformally Soft Photons and Gravitons, JHEP 01 (2019) 184 [arXiv: 1810.05219] [INSPIRE].

[7] S. Stieberger and T.R. Taylor, Symmetries of Celestial Amplitudes, Phys. Lett. B 793 (2019) 141 [arXiv: 1812.01080] [INSPIRE].

[8] E. Himwich and A. Strominger, Celestial current algebra from Low's subleading soft theorem, Phys. Rev. D 100 (2019) 065001 [arXiv: 1901.01622] [INSPIRE].

[9] W. Fan, A. Fotopoulos and T.R. Taylor, Soft Limits of Yang-Mills Amplitudes and Conformal Correlators, JHEP 05 (2019) 121 [arXiv: 1903.01676] [INSPIRE].

[10] M. Pate, A.-M. Raclariu and A. Strominger, Conformally Soft Theorem in Gauge Theory, Phys. Rev. D 100 (2019) 085017 [arXiv: 1904.10831] [INSPIRE]. 
[11] T. Adamo, L. Mason and A. Sharma, Celestial amplitudes and conformal soft theorems, Class. Quant. Grav. 36 (2019) 205018 [arXiv:1905.09224] [INSPIRE].

[12] D. Nandan, A. Schreiber, A. Volovich and M. Zlotnikov, Celestial Amplitudes: Conformal Partial Waves and Soft Limits, JHEP 10 (2019) 018 [arXiv:1904.10940] [InSPIRE].

[13] A. Puhm, Conformally Soft Theorem in Gravity, JHEP 09 (2020) 130 [arXiv:1905.09799] [INSPIRE].

[14] M. Pate, A.-M. Raclariu, A. Strominger and E.Y. Yuan, Celestial operator products of gluons and gravitons, Rev. Math. Phys. 33 (2021) 2140003 [arXiv:1910.07424] [InSPIRE].

[15] A. Guevara, Notes on Conformal Soft Theorems and Recursion Relations in Gravity, arXiv: 1906.07810 [INSPIRE].

[16] A. Fotopoulos, S. Stieberger, T.R. Taylor and B. Zhu, Extended BMS Algebra of Celestial CFT, JHEP 03 (2020) 130 [arXiv:1912.10973] [InSPIRE].

[17] A. Fotopoulos, S. Stieberger, T.R. Taylor and B. Zhu, Extended Super BMS Algebra of Celestial CFT, JHEP 09 (2020) 198 [arXiv:2007.03785] [INSPIRE].

[18] H. Jiang, Celestial superamplitude in $\mathcal{N}=4$ SYM theory, JHEP 08 (2021) 031 [arXiv:2105.10269] [INSPIRE].

[19] A. Brandhuber, G.R. Brown, J. Gowdy, B. Spence and G. Travaglini, Celestial superamplitudes, Phys. Rev. D 104 (2021) 045016 [arXiv:2105.10263] [InSPIRE].

[20] Y. Hu, L. Ren, A.Y. Srikant and A. Volovich, Celestial Dual Superconformal Symmetry, MHV Amplitudes and Differential Equations, arXiv:2106.16111 [INSPIRE].

[21] N. Arkani-Hamed, Y. Bai and T. Lam, Positive Geometries and Canonical Forms, JHEP 11 (2017) 039 [arXiv : 1703.04541] [inSPIRE].

[22] A. Sharma, Ambidextrous light transforms for celestial amplitudes, arXiv:2107.06250 [INSPIRE].

[23] N. Arkani-Hamed, F. Cachazo, C. Cheung and J. Kaplan, A Duality For The S Matrix, JHEP 03 (2010) 020 [arXiv:0907.5418] [inSPIRE].

[24] L.J. Mason and D. Skinner, Dual Superconformal Invariance, Momentum Twistors and Grassmannians, JHEP 11 (2009) 045 [arXiv:0909.0250] [INSPIRE].

[25] N. Arkani-Hamed and J. Trnka, The Amplituhedron, JHEP 10 (2014) 030 [arXiv: 1312.2007] [INSPIRE].

[26] J.M. Drummond, J.M. Henn and J. Plefka, Yangian symmetry of scattering amplitudes in $N=4$ super Yang-Mills theory, JHEP 05 (2009) 046 [arXiv:0902.2987] [INSPIRE].

[27] J.M. Drummond and L. Ferro, Yangians, Grassmannians and T-duality, JHEP 07 (2010) 027 [arXiv: 1001.3348] [INSPIRE].

[28] J.M. Drummond and L. Ferro, The Yangian origin of the Grassmannian integral, JHEP 12 (2010) 010 [arXiv: 1002.4622] [INSPIRE].

[29] R. Britto, F. Cachazo and B. Feng, New recursion relations for tree amplitudes of gluons, Nucl. Phys. B 715 (2005) 499 [hep-th/0412308] [INSPIRE].

[30] R. Britto, F. Cachazo, B. Feng and E. Witten, Direct proof of tree-level recursion relation in Yang-Mills theory, Phys. Rev. Lett. 94 (2005) 181602 [hep-th/0501052] [INSPIRE]. 